\begin{tabular}{|l|l|l|l|l|l|}
\hline $\begin{array}{l}\text { Nereis. Revista Iberoamericana } \\
\begin{array}{l}\text { Interdisciplinar de Métodos, } \\
\text { Modelización y Simulación }\end{array}\end{array}$ & 13 & $73-83$ & $\begin{array}{c}\text { Universidad Católica de } \\
\text { Valencia San Vicente Mártir }\end{array}$ & $\begin{array}{c}\text { Valencia } \\
\text { (España) }\end{array}$ & \begin{tabular}{l} 
ISSN 1888-8550 \\
\hline
\end{tabular}
\end{tabular}

\title{
Modelo experimental de queratosis actínica por exposición a radiación ultravioleta $\mathrm{A}$
}

\author{
Experimental model of actinic keratosis due to exposure to ultraviolet A radiation
}

Fecha de recepción y aceptación: 17 de marzo de 2021 y 22 de abril de 2021

DOI: $10.46583 /$ nereis_2021.13.841

\author{
Liuba Peñate Alvariño ${ }^{1}$, Grety Margarita Garau ${ }^{2}$, Arianna Valido Díaz ${ }^{1}$, Arletis Ferrer Pérez ${ }^{2}$, Yisel

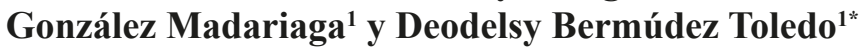 \\ ${ }^{1}$ Unidad de Toxicología Experimental. Universidad de Ciencias Médicas de Villa Clara. Cuba \\ ${ }^{2}$ Hospital Provincial "Arnaldo Milián Castro". Villa Clara. Cuba \\ *Correspondencia: Universidad de Ciencias Médicas de Villa Clara. Unidad de Toxicología Experimental. Calle $7 \mathrm{ma} \mathrm{\#} 80$ e/ prolon- \\ gación de Colón y Hospital Arnaldo Milián Castro. 50100 Villa Clara. Cuba.E-mail: deodelsybt@infomed.sld.cu
}

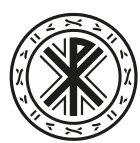

Universidad Católica de Valencia San Vicente Mártir

\section{RESUMEN}

Las queratosis actínicas son lesiones cutáneas preneoplásicas frecuentes causadas por una exposición crónica a las radiaciones solares. La obtención de modelos animales que contribuyan a comprender los mecanismos implicados en esta patología y a evaluar nuevas terapias farmacológicas resulta de gran interés para la comunidad científica. Se desarrolló un biomodelo de queratosis actínica en ratones Balb/c, empleando como inductor la radiación UVA. Se aplicaron dos esquemas de irradiación: Grupo I, expuesto a tres sesiones diarias de radiación de 2 h y Grupo II, dos sesiones diarias de $4 \mathrm{~h}$, con igual tiempo de receso entre sesiones y durante un período de 14 días. Se evaluaron parámetros macroscópicos y microscópicos, y la frecuencia de aparición de micronúcleos en sangre periférica. Los animales correspondientes al Grupo I no mostraron lesiones mascroscópicas y microscópicamente evidenciaron una leve acantosis con ausencia de displasia; mientras que en los animales del Grupo II se observaron lesiones macroscópicas que se correlacionan histopatológicamente con la presencia de una queratosis actínica. La frecuencia de micronúcleos obtenida en el Grupo II fue significativamente superior a la formación de micronúcleos espontáneos en el Grupo Control. El biomodelo experimental de queratosis actínica obtenido reproduce el efecto de las radiaciones ultravioletas sobre la piel.

PALABRAS CLAVE: queratosis solar, lesión preneoplásica, ratones Balb/c.

\section{ABSTRACT}

Actinic keratoses are frequent preneoplastic skin lesions caused by chronic exposure to solar radiation. Obtaining animal models that contribute to understanding the mechanisms involved in this pathology and evaluating new pharmacological therapies is of great interest to the scientific community. A biomodel of actinic keratosis was developed in Balb/c mice, using UVA radiation as an inducer. Two irradiation schemes were applied: Group I, exposed to three daily radiation sessions of $2 \mathrm{~h}$ and Group II, two daily sessions of $4 \mathrm{~h}$, with the same break time between ses- 
sions and for a period of 14 days. Macroscopic and microscopic parameters were evaluated, as well as the frequency of appearance of micronuclei in peripheral blood. The animals corresponding to Group I did not show mascroscopic lesions and microscopically they showed a slight acanthosis with the absence of dysplasia; while in Group II animals macroscopic lesions were observed that histopathologically correlate with the presence of an actinic keratosis. The frequency of micronuclei obtained in Group II was significantly higher than the formation of spontaneous micronuclei in the Control Group. The experimental biomodel of actinic keratosis obtained reproduces the effect of ultraviolet radiation on the skin.

KEYWORDS: solar keratosis, preneoplastic lesion, Balb/c mice.

\section{INTRODUCCIÓN}

El cáncer de piel es una de las neoplasias más comunes y su incidencia ha incrementado en las últimas décadas [1]. Los principales tipos de cáncer de piel son melanoma y no melanoma, en este último grupo se enmarcan las lesiones premalignas (queratosis actínicas, QA) y malignas más frecuentes en dermatología, el carcinoma basocelular (CBC) y el carcinoma de células escamosas (CCE) [2,3].

Las QA-también referidas como queratosis senil o queratosis solar-son lesiones preneoplásicas caracterizadas por una atipia variable de los queratinocitos [4,5]. Pertenece a las lesiones cutáneas más comunes, siendo uno de los motivos de consultas más frecuentes en la práctica clínica dermatológica [6,7]. Su prevalencia es muy variable entre los diferentes países con un aumento dependiente de la edad [6,8], por lo que se espera un incremento gradual en su frecuencia con el envejecimiento global de la población. La QA puede evolucionar a CCE invasivo y aunque la probabilidad de transición de una sola lesión parece ser baja, aumenta rápidamente en presencia de QA múltiple, cancerización de campo o inmunosupresión [5,9].

La exposición crónica a las radiaciones solares constituye el factor principal involucrado en su patogénesis. Su componente UV actúa como iniciador y promotor de la carcinogénesis en la QA [10]. Las células cutáneas pueden resultar dañadas directamente por la radiación ultravioleta (RUV) o indirectamente mediada por la sobreproducción de especies reactivas de oxígeno (ERO) $[11,12]$. Las mutaciones del gen supresor de tumores TP53 inducidas por RUV son de gran importancia en el desarrollo de la QA [13,14].

En nuestro estudio fue empleada la radiación UVA (320-400 nm) basado en que más de un $90 \%$ de la radiación que llega a la superficie de la Tierra está compuesta por rayos UVA y al poseer las mayores longitudes de onda del espectro UV y baja energía, tiene mayor capacidad de atravesar la epidermis hasta niveles profundos de la dermis [15]. La radiación UVA induce estrés fotooxidativo, debido a la generación de ERO que producen daños en el ADN celular, principalmente los dímeros de pirimidina ciclobutano (cyclobutane pyrimidine dimer, CPD) [16], los cuales causan mutaciones conocidas como huellas genéticas UV [17] que desencadenan procesos inflamatorios y causan inmunosupresión y cáncer de piel.

Teniendo en cuenta la prevalencia de QA en los últimos años y su potencial de progresión a CCE invasivo $[9,18]$ resulta importante el desarrollo de modelos experimentales que contribuyan a la evaluación de nuevas terapias farmacológicas. En nuestro trabajo nos propusimos obtener un modelo 
animal de QA por exposición a la radiación UVA, mediante la evaluación de parámetros macroscópicos y microscópicos, y de la frecuencia de aparición de micronúcleos en sangre periférica como indicador de daño en el material genético.

\section{MATERIAL Y MÉTODOS}

\section{Animales}

El protocolo experimental fue evaluado y aprobado por el Comité de Ética Animal de la Unidad de Toxicología Experimental de la Universidad Médica de Villa Clara, en Cuba, de acuerdo con las Guías ARRIVE [19]. Se utilizaron ratones machos Balb/c como modelo biológico de 20 a 25 g y de 6 a 8 semanas de edad procedentes del Centro Nacional para la Producción de Animales de Laboratorio (CENPALAB) en La Habana. Los animales se mantuvieron en jaulas de PVC con cubierta metálica de rejas y en condiciones libres de patógenos específicos, con acceso al agua y alimento (pienso convencional para roedores) ad libitum, durante todo el experimento, así como a un régimen de luz-oscuridad de $12-12$ horas y temperatura de $22 \pm 2{ }^{\circ} \mathrm{C}$.

\section{Diseño experimental}

Tres grupos experimentales de 4 animales cada uno fueron conformados (control negativo y dos grupos irradiados). Para la irradiación fue empleada una lámpara UVA con una irradiancia de 295-390 nm, aplicada 5 días/semana durante un período de 14 días. Para ello, los animales fueron situados en jaulas de PVC con separadores individuales y una cubierta de celosía metálica y colocadas bajo la lámpara de luz UV a una distancia foco/piel de $20 \mathrm{~cm}$ [20,21]. Las dimensiones del área donde se encontraba el animal durante la radiación y la distancia aplicada de la lámpara UV permitieron la exposición uniforme de todos los animales, teniendo en cuenta que las orejas sería la zona de mayor absorción de irradiación, es decir la zona sobreexpuesta a la RUV debido a la ausencia de pelaje y por tanto la más susceptible de desarrollar un daño actínico.

Fueron aplicados 2 esquemas de irradiación de manera que los grupos experimentales quedaron distribuidos de la siguiente forma: Grupo 0: Control negativo, no recibió irradiación, Grupo I: tiempo de exposición de tres sesiones diarias de $2 \mathrm{~h}$ cada una, con un receso entre ellas de $30 \mathrm{~min}$ (3S:2h c/1/2h), Grupo II: tiempo de exposición de dos sesiones diarias de $4 \mathrm{~h}$ cada una, con un receso entre ambas de $30 \mathrm{~min}(2 \mathrm{~S}: 4 \mathrm{~h} \mathrm{c} / 1 / 2 \mathrm{~h})$.

Los esquemas de irradiación propuestos estuvieron basados en estudios previos de nuestro equipo de investigación, en los cuales con la aplicación de menores tiempos de exposición a la radiación, no se generaron lesiones cancerígenas ni precancerígenas en nuestras condiciones experimentales. 


\section{Estudio macroscópico}

Se realizaron observaciones diarias con el objetivo de cuantificar y caracterizar las lesiones macroscópicas. Las principales lesiones macroscópicas evaluadas fueron la presencia de eritema, congestión, aumento de la vascularización, telangiectasias, vesículas (o ampollas), presencia de lesiones costrosas, queratósicas y ulcerosas.

\section{Estudio microscópico}

Concluido el período de irradiación, los animales fueron sacrificados mediante una sobredosis de anestésico inhalado, acorde a las recomendaciones para la eutanasia de los animales de experimentación [22].

A continuación, se realizó la toma de muestra para un estudio histopatológico de la piel de las orejas. Se seleccionó la porción superior del epitelio auricular de los animales correspondientes a los diferentes grupos experimentales. Las muestras colectadas fueron fijadas en formol neutro tamponado al $10 \%$ al menos durante $48 \mathrm{~h}$, incluidas en parafina, seccionadas a $5 \mu \mathrm{m}$ y teñidas con hematoxilina-eosina. Fueron evaluados como criterios histológicos la presencia de epitelio normal, displasia epitelial, carcinoma in situ y queratosis actínica, mediante microscopía de luz (Olimpus BX51TRF, Japón, objetivo 40X) acoplado a una cámara digital (Olimpus E330-ADU 1-2X, Japón).

\section{Ensayo de micronúcleos en sangre periférica}

El ensayo de micronúcleos $(\mathrm{MN})$ se considera un protocolo práctico universalmente validado y técnicamente factible, útil para evaluar daños citogenéticos inducidos por agentes genotóxicos.

La muestra de sangre se obtuvo mediante el procedimiento del tijereteado de la cola del ratón luego de finalizada la última sesión de irradiación y antes del sacrificio. Se tomaron $5 \mu$ y colocados sobre la lámina previamente preparada con naranja de acridina. Fue registrada la frecuencia de los reticulocitos con micronúcleos (MNRET) sobre la base de 1000 reticulocitos por láminas. La observación se realizó en un microscopio de fluorescencia con la combinación de un filtro azul excitatorio de $480 \mathrm{~nm}$ y un filtro de barrera amarillo-naranja de $515 \mathrm{~nm}$.

La variable evaluada fue la frecuencia basal de $\mathrm{MN}$ en reticulocitos de sangre periférica de ratones Balb/c (grupo control negativo) y la frecuencia de MN inducida postexposición a la radiación UV, solo en el grupo irradiado que mostró alteraciones macroscópicas y microscópicas.

\section{Análisis estadístico}

Los resultados obtenidos en la evaluación de los parámetros cuantitativos (cantidad de MNRET) fueron comparados estadísticamente entre los grupos. Para el procesamiento descrito se empleó el paquete estadístico SPSS para Windows Versión 20.0. Se procedió a verificar la normalidad en la distribución con el empleo de la prueba de Shapiro-Wilks y la prueba de Levene para homogeneidad de 
varianzas. Posteriormente se aplicó la prueba $t$-student para evaluar las diferencias entre grupos, con un nivel de significación $\mathrm{p}<0,05$.

\section{RESULTADOS Y DISCUSIÓN}

\section{Estudio macroscópico}

Durante la realización del experimento todos los animales, tras su exposición a la radiación, presentaron lesiones macroscópicas con una evolución diferente relacionada con la radiación acumulada. Todos los animales al ser expuestos a la radiación mostraron un leve enrojecimiento en la zona de las orejas, siendo reversible pasados $60 \mathrm{~min}$ postexposición en los animales correspondientes al Grupo I (3S:2h c/1/2h). Sin embargo, en el $100 \%$ de los animales del Grupo II (2S: $4 \mathrm{~h} \mathrm{c} / 1 / 2 \mathrm{~h}$ ) se evidenció un eritema permanente con un incremento de la vascularización y congestión, además de desarrollar lesiones blanquecinas con presencia de queratosis que se localizaban sobre las áreas de aspecto eritematoso y que aumentaban progresivamente de tamaño en la parte interna del lóbulo de la oreja (figura 1).

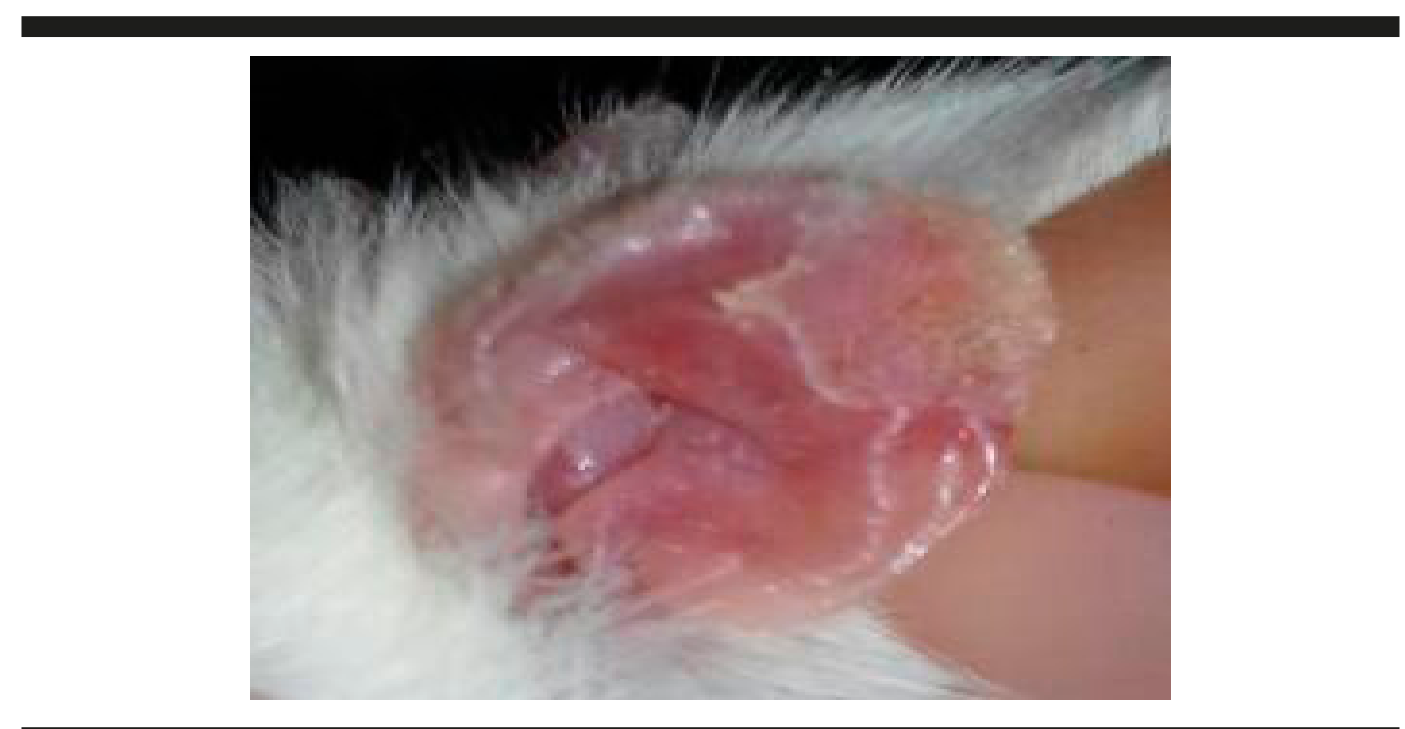

Fig. 1. Lesiones macroscópicas desarrolladas en la parte interna de las orejas de los ratones del Grupo II (2S: $4 \mathrm{~h}$ c / 1 $1 / 2 \mathrm{~h})$

Los aspectos clínicos observados en nuestro biomodelo experimental se correlacionan con las características clínicas descritas para las QA desarrolladas en humanos. Esta lesión preneoplásica se presenta como pápulas, parches o placas escamosas eritematosas a pardo rojizas, adherentes, ásperas y secas que se desarrolla en la piel expuesta crónicamente al sol y pueden presentarse como lesión única o múltiples lesiones [4,23].

La localización de las lesiones observadas puede deberse esencialmente a la ausencia de pelaje en esta zona, lo que la hace más susceptible al daño debido a una mayor energía total absorbida. La piel 
pubescente es una barrera importante para las alteraciones provocadas por factores externos como las radiaciones solares, lo que explica la localización de las QA generalmente en zonas de la piel sobreexpuestas a la RUV (cuero cabelludo con calvicie en varones, cara, frente, mejillas, nariz, pabellones auriculares, cuello, dorso de las manos y antebrazos, piernas en mujeres) [4,23].

En nuestro estudio, la aparición de alteraciones macroscópicas en las orejas de los animales del Grupo II (2S: 4h c/1 $\left.\frac{1}{2} \mathrm{~h}\right)$, en contraste con los animales del Grupo I (3S:2h c/1/2h), pudiera deberse al esquema de irradiación aplicado. Es importante destacar que el efecto de la RUV es dependiente de la energía $\left(\mathrm{J} / \mathrm{cm}^{2}\right)$ total recibida y que la exposición solar acumulada a la radiación UV es uno de los predictores potentes de riesgo de QA. El esquema de irradiación aplicado a los ratones del Grupo II ( $2 \mathrm{~S}: 4 \mathrm{~h} \mathrm{c} / 1 \frac{1}{2} \mathrm{~h}$ ) incluyó un mayor tiempo de exposición a la RUV y de manera continua, recibiendo los animales una energía superior en cada sesión respecto a los del Grupo I ( $\left.3 \mathrm{~S}: 2 \mathrm{~h} \mathrm{c} / 1 \frac{1}{2} \mathrm{~h}\right)$.

Partiendo de que la exposición acumulada a la luz UV o a la luz solar actúa como un iniciador y promotor de la carcinogénesis en la QA [10] es de esperar que progresivamente el daño solar acumulado provoque inestabilidad a nivel genómico, lo que resulta en la inhibición o sobreexpresión de genes que regulan la proliferación celular y la apoptosis, lo cual conduce a una alteración de los mecanismos de reparación.

\section{Estudio microscópico}

Clínicamente no existe un umbral claro entre las QA y los CCE. La QA se considera parte del espectro evolutivo del CCE, descrita como un CCE “embrionario". Por tanto, el diagnóstico histopatológico constituye una herramienta importante en la confirmación y evaluación de la progresión de esta patología.

Durante el estudio los animales pertenecientes al Grupo Control mantuvieron una piel sana, lo que se correlaciona con las características microscópicas obtenidas para un epitelio normal: superficie epitelial delgada, asociada con folículos pilosos y glándulas sebáceas, soportadas por tejido conectivo y adiposo (figura $2 \mathrm{a})$.

El análisis microscópico obtenido para el Grupo I ( $3 \mathrm{~S}: 2 \mathrm{~h}$ c/1/2h) evidenció la presencia de acantosis leve con ausencia de displasia en el $50 \%$ de los animales (figura 2b), mientras que el $100 \%$ de los animales del Grupo II ( $\left.2 \mathrm{~S}: 4 \mathrm{~h} \mathrm{c} / \frac{1}{2} \mathrm{~h}\right)$ desarrollaron alteraciones histopatológicas notablemente diferentes respecto al Grupo Control (epitelio normal). Los desórdenes tisulares observados se describieron como engrosamiento de la capa superficial de queratina (hiperqueratosis), presencia de acantosis en epidermis, pérdida de polaridad, ensanchamiento de los espacios intercelulares, diplasia epitelial con núcleos agrandados y aumento de la picnosis nuclear, incremento en la relación núcleo/ citoplasma, presencia de figuras mitóticas y denso infiltrado inflamatorio caracterizado por una gran cantidad de linfocitos en dermis (figura 2c). Las distorsiones graduales de la arquitectura normal del tejido, observadas en el epitelio auricular de los animales pertenecientes al Grupo II (2S: 4h c/1/2h), fueron diagnosticadas como queratosis actínica. 


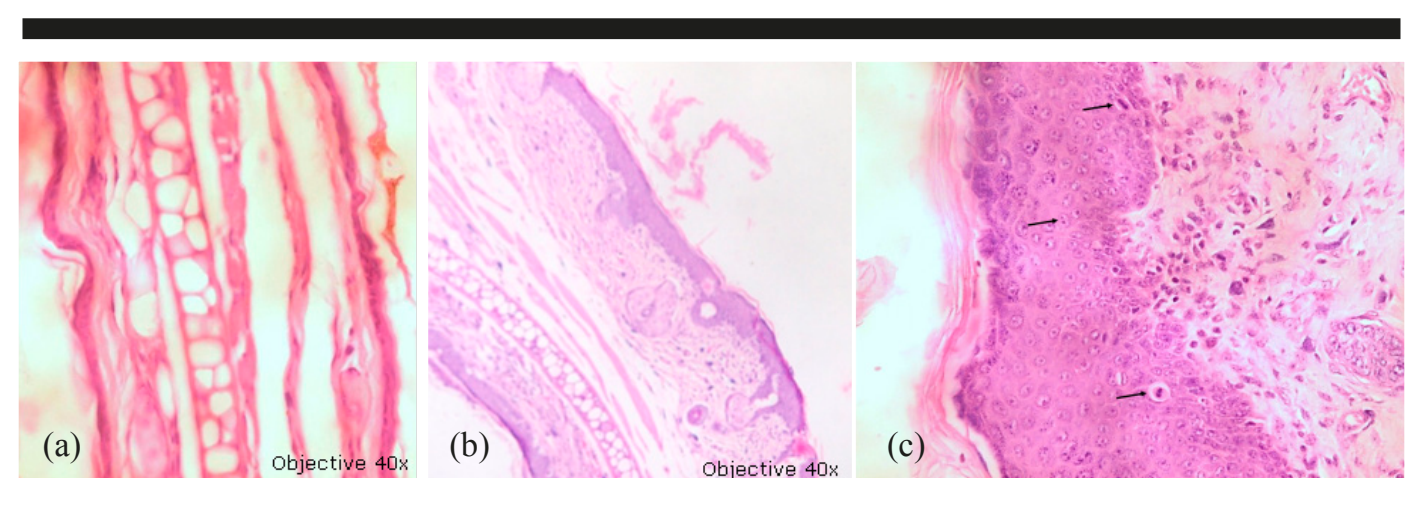

Fig. 2. Sección histológica de la piel de las orejas de los ratones objeto de estudio. (a) Grupo control negativo. (b) Grupo I (3S: $2 \mathrm{~h} \mathrm{c} \mathrm{/} \mathrm{1/2h).} \mathrm{La} \mathrm{flecha} \mathrm{indica} \mathrm{el} \mathrm{epitelio}$ ligeramente aumentado. (c) Alteraciones histopatológicas desarrolladas en el epitelio auricular de los ratones correspondientes al Grupo II (2S: 4h c / 1/2h). Las flechas indican células en diferentes fases mitóticas. Fuente: elaboración propia.

La QA es una neoplasia intraepidérmica temprana resultante de la proliferación anormal de queratinocitos epidérmicos atípicos [4,5]. Las alteraciones citológicas desarrolladas en esta lesión incluyen pérdida de polaridad, agrandamiento nuclear y pleomorfismo, maduración desregulada, aumento del número de mitosis, hiperqueratosis y acantosis [4,5], lo cual se correlaciona con los resultados citológicos obtenidos en los animales pertenecientes al Grupo II ( $2 \mathrm{~S}$ : $\left.4 \mathrm{~h} \mathrm{c} / 1 \frac{1}{2} \mathrm{~h}\right)$ del presente estudio.

Las alteraciones histopatológicas citadas anteriormente apoyan la hipótesis de que las QA tienen características de malignidad desde su génesis, desde el punto de vista de los cambios citológicos que presentan los queratinocitos epidérmicos, que son similares a los observados en los CCE [9,18], incluyendo las mutaciones idénticas en la proteína p53 a nivel molecular [13,14,24]. Es por ello que aunque clásicamente son categorizadas como lesiones preneoplásicas, algunos autores sugieren considerarlas como neoplasias in situ, ya que derivan de modificaciones clonales del ADN en los queratocitos $[9,18,25]$. La dificultad para establecer criterios inequívocos para determinar cuándo una QA sufre una transformación de CCE refuerza esta hipótesis.

El efecto acumulativo de la dosis de radiación aplicada a los animales del Grupo II (2S: 4h c/1/2h) pudiera ser la variable principal relacionada a las irregularidades histológicas observadas en este grupo. En el Grupo I (3S: $2 \mathrm{~h}$ c / 1 1/2 h), donde las dosis de radiación fueron más fraccionadas, se evidenció menor daño respecto al Grupo II ( $2 \mathrm{~S}$ : $\left.4 \mathrm{~h} \mathrm{c} / \frac{1}{2} \mathrm{~h}\right)$, en este último el esquema de irradiación incluyó un mayor tiempo de exposición continua y menor división de la dosis. En la medida en que aumenta el daño por la exposición repetida a las RUV, los mecanismos celulares existentes para reparar el daño del ADN o para inducir apoptosis con el objetivo de eliminar las células severamente dañadas son poco eficientes debido a la reacción molecular en cadena que provocan las RUV en la dermis, que resultan en la desorganización del colágeno y de la elastina, así como otros componentes de la matriz extracelular dérmica [26]. Es por ello que la exposición repetida a la RUV hace que la reparación de la matriz dérmica sea más deficiente. El efecto acumulativo de modificaciones no reparadas puede desencadenar la aparición de mutaciones puntuales en genes diana como el gen supresor de tumores p53 [27]. Los queratinocitos basales con el gen supresor de tumores TP53 mutado no responden 
normalmente a la apoptosis inducida por UV, lo que permite una mayor proliferación y el desarrollo de nuevas anomalías genéticas [27].

Los rayos UVA producen daños en el ADN, principalmente los CPD [16], los cuales causan mutaciones conocidas como huellas genéticas UV [17]. Si el nivel de los CPD en la célula supera su capacidad para repararlos, y estos persisten durante la fase $\mathrm{S}$ del ciclo celular, una polimerasa propensa a error puede polimerizar estos fotoproductos [28] y producir las ya mencionadas huellas genéticas UV. Cuando estas mutaciones se acompañan de la pérdida de la función de los genes supresores de tumores, como el de la proteína p53, la célula pierde la capacidad de reparar el daño, lo que da lugar a procesos de expansión clonal de las células mutantes involucradas en las fases iniciales del cáncer de piel. Las mutaciones del gen supresor de tumores TP53 inducidas por RUV son de gran importancia en el desarrollo de la QA [14,24].

\section{Ensayo de micronúcleos en sangre periférica}

Los MN son masas de cromatina en el citoplasma que forman pequeños núcleos "micronúcleo" visibles al microscopio óptico. Se originan a partir de fragmentos cromosómicos o cromosomas enteros que durante el proceso de división celular quedan retrasados en la fase de segregación cromosómica (anafase), debido a errores durante la replicación, la posterior división del ADN, roturas cromosómicas o efectos propios de la radiación y sustancias genotóxicas; es así que no terminan siendo incorporados al núcleo de la célula hija [29,30].

El ensayo de MN realizado a los animales correspondientes al Grupo II (2S: 4h c/1/2h) arrojó una frecuencia de aparición de MN tres veces superior a la formación de MN espontáneos en el Grupo Control Negativo (figura 3), siendo esta variable un indicador de daño en el material genético. La prueba $t$-student aplicada a los valores obtenidos mostró diferencias significativas $(\mathrm{p}<0,05)$ entre ambos grupos experimentales. Los reticulocitos en sangre periférica fueron observados con una fluorescencia roja debido a la presencia de ARN ribosomal en estos, mientras que las células nucleadas normales mostraron una fluorescencia parda oscura. Los MN mostraron una coloración fluorescente verde-amarilla.

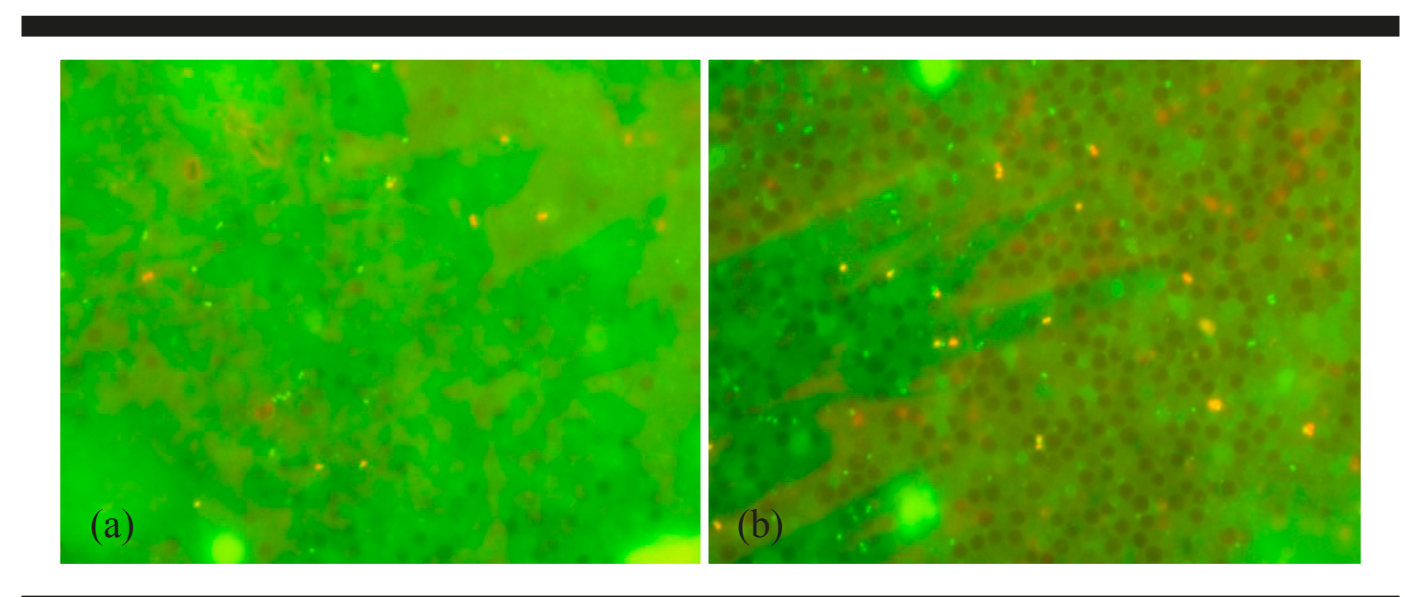

Fig. 3. Micronúcleos en reticulocitos de sangre periférica teñidos con naranja de acridina

(a) Grupo control negativo (b) Grupo II (2S: 4h c / 1 1/2h). Fuente: elaboración propia. 
El aumento de la frecuencia de MNRET observado en el Grupo II (2S: 4h c/1/2h) respalda el criterio del efecto que tienen las RUV sobre el material genético. En el esquema de radiación aplicado a este grupo de animales la energía total recibida durante el estudio y el tiempo de exposición son variables determinantes en la generación de alteraciones en el ADN. Los resultados obtenidos en este ensayo están en correspondencia con las alteraciones histopatológicas observadas en este grupo. En consonancia con nuestros resultados, Zúñiga et al. [31] obtuvieron un aumento de eritrocitos micronucleados en sangre periférica de ratas expuestas a radiación UVA, y concluyeron que la exposición a la luz UVA induce un incremento en el número de estos pequeños núcleos con daño aparente en la piel.

La fisiopatología de la piel fotodañada demuestra que la QA es la manifestación inicial de la carcinogénesis inducida por la radiación solar. La predisposición de las células a una inestabilidad cromosómica (traslocaciones cromosómicas) y a un aumento de la probabilidad de alteraciones genómicas explica la acción iniciadora o promotora carcinogénica de la RUV [10,31]. La Agencia de Investigación del Cáncer de la OMS, incluyó a las RUV en el grupo 1 de agentes carcinógenos [32].

El daño causado al ADN por la RUV puede ser producto de la absorción directa de la energía de los fotones o del daño indirecto donde los cromóforos endógenos transfieren la carga a otras moléculas que causan modificaciones en el ADN. El daño directo más común que se origina son los $\mathrm{CPD}$, que representan aproximadamente las tres cuartas partes de los fotoproductos. Su forma más frecuente son los dímeros de timina (TT); este fotoproducto, además de ser el más abundante, es de reparación lenta y mutagenicidad distintiva $[12,16]$.

\section{CONCLUSIONES}

En nuestro estudio, el esquema de irradiación aplicado al Grupo II ( $2 \mathrm{~S}: 4 \mathrm{~h} \mathrm{c} / 1 / 2 \mathrm{~h})$ generó lesiones macroscópicas y microscópicas en la piel de los animales objeto de estudio. Se diagnosticó histopalógicamente una QA, descrita como una lesión preneoplásica con potencialidades para evolucionar a un CCE invasivo. Este esquema de irradiación también provocó un incremento de la frecuencia de $\mathrm{MN}$, indicador de daño genotóxico. Por tanto, el esquema de irradiación consistente en dos sesiones diarias de $4 \mathrm{~h}$, con igual tiempo de receso entre sesiones y durante un período de 14 días, permitió la obtención de un biomodelo experimental de QA que reproduce las alteraciones macroscópicas y microscópicas descritas para esta lesión cutánea en humanos.

\section{REFERENCIAS BIBLIOGRÁFICAS}

[1] Bray F, Ferlay J, Soerjomataram I, Siegel RL, Torre LA, Jemal A. Global cancer statistics 2018: GLOBOCAN estimates of incidence and mortality worldwide for 36 cancers in 185 countries. CA Cancer J Clin. 2018;68(6):394-424.

[2] John SM, Trakatelli M, Gehring R, Finlay K, Fionda C, Wittlich M, et al. CONSENSUS REPORT: Recognizing non-melanoma skin cancer, including actinic keratosis, as an occupational disease-A Call to Action. J Eur Acad Dermatol Venereol. 2016;30(Suppl. 3):38-45. 
[3] Vimercati L, De Maria L, Caputi A, Silvana CE, Mansi F, Cavone D, et al. Non-Melanoma Skin Cancer in Outdoor Workers: A Study on Actinic Keratosis in Italian Navy Personnel. Int J Environ Res Public Health. 2020;17(7):2321. doi: 10.3390/ijerph17072321. PMID: 32235587; PMCID: PMC7177289.

[4] Schmitz L, Kahl P, Majores M, Bierhoff E, Stockfleth E, Dirschka T. Actinic keratosis: correlation between clinical and histological classification systems. J Eur Acad Dermatol Venereol. 2016;30(8):1303-07.

[5] Filosa A, Filosa G. Actinic keratosis and squamous cell carcinoma: clinical and pathological features. G Ital Dermatol Venereol. 2015;150(4):379-84.

[6] Ferrándiz C, Plazas MJ, Sabaté M, Palomino R, EPIQA Study Group. Prevalence of actinic keratosis among dermatology outpatients in Spain. Actas Dermosifiliogr. 2016;107(8): 674-80.

[7] Prieto HC, Marchiori BR. Actinic keratoses: review of clinical, dermoscopic, and therapeutic aspects. An Bras Dermatol. 2019;94(6):637-57.

[8] Hensen P, Müller ML, Haschemi R, Ständer H, Luger TA, Sunderkötter C, et al. Predisposing factors of actinic keratosis in a North-West German population. Eur J Dermatol. 2015;19(4):345-54.

[9] Schmitz L, Gambichler T, Kost C, Gupta G, Stucker M, Stockfleth E, et al. Cutaneous squamous cell carcinomas are associated with basal proliferating actinic keratoses. Br J Dermatol. 2019; 180(4):916-21.

[10] Zaeem A, Sultan AM, Maqbool A, Ellahi BM, Hussain T. UV radiations from sunlight as a potential carcinogen for the development of skin malignancy: A review of the current status and future prospects. Biomedical Letters. 2019;5(2):79-87.

[11] Xian D, Lai R, Song J, Xiong X, Zhong J. Emerging Perspective: Role of Increased ROS and Redox Imbalance in Skin Carcinogenesis. Oxidative Medicine and Cellular Longevity. 2019(3):111, ID 8127362. doi: 10.1155/2019/8127362. PMID: 31636809; PMCID: PMC6766104

[12] Van de Glind G, Rebel H, Van Kempen M, Tensen K, De Gruijl F. Fractionation of a tumorinitiating UV dose introduces DNA damage-retaining cells in hairless mouse skin and renders subsequent TPA-promoted tumors non-regressing. Oncotarget. 2016;7: 8067-77.

[13] Bakshi A, Shafi R, Nelson J, Cantrell WC, Subhadarshani S, Andea A, et al. The clinical course of actinic keratosis correlates with underlying molecular mechanisms. Br J Dermatol. 2020;182(4):995-1002.

[14] Heerfordt IM, Nissen CV, Poulsen T, Philipsen PA, Wulf HC. Thickness of actinic keratosis does not predict dysplasia severity or P53 expression. Sci Rep. 2016;6:33952. doi: 10.1038/ srep33952. PMID: 27670104; PMCID: PMC5037398.

[15] Gegotek A, Bielawska K, Biernacki M, Dobrzyńska I, Skrzydlewska E. Time-dependent effect of rutin on skin fibroblasts membrane disruption following UV radiation. Redox Biology. 2017;12:733-44.

[16] Tewari A, Sarkany RP, Young AR. UVA induces cyclobutane pyrimidine dimers but not 6-4 photoproducts in human skin in vivo. J. Invest. Dermatol. 2012;132:394-400.

[17] Brash DE. UV signature mutations. Photochem Photobiol. 2015;91(1):15-26.

[18] Schmitz L, Grinblat B, Novak B, Hoeh AK, Handschke K, Dobbeler CV, et al. Somatic mutations in kinetochore gene KNSTRN are associated with basal proliferating actinic keratoses and cutaneous squamous cell carcinoma. J Eur Acad Dermatol Venereol. 2019;33(8):1535-40. 
[19] McGrath JC, Drummond GB, McLachlan EM, Kilkenny C, Wainwright CL. Guidelines for reporting experiments involving animals: the ARRIVE guidelines. British Journal of Pharmacology. 2010;160(7):1573-1576.

[20] Cano GA, Gómez GF, Álvarez SN, Sánchez-Pedreño GP, Vicente OV. Modelo de fotocarcinogénesis cutánea en ratones SKH-1 por radiación ultravioleta. Rev Esp Patol. 2010;43(4):191-95.

[21] Gómez GF, López LA, Guerrero SY, Sánchez SM, Martínez DF, Camacho AF. Chemopreventive effect of pomegranate and cocoa extracts on ultraviolet radiation induced photocarcinogenesis in SKH-1 mice. PLoSONE. 2020;15(4):e0232009. doi: 10.1371/journal.pone.0232009. PMID: 32353018; PMCID: PMC7192448.

[22] Close B, Banister K, Baumans V, Bernoth E, Bromage N, Bunyan J, et al. Recommendations for the euthanasia of experimental animals. Lab Animal. 1997;31(1),1-32.

[23] Schmitt VJ, Miot HA. Actinic keratosis: a clinical and epidemiological revision. An Bras Dermatol. 2012;87(3):425-34.

[24] Li X, Li Z, Han Z, Zhang L, Liu Z, Liu B. Gene Expression Patterns of Cutaneous Squamous Cell Carcinoma and Actinic Keratosis: Biomarkers Screening for Skin Disease Diagnosis. Ann Dermatol. 2018;30(5):536-43.

[25] Yoshifuku A, Fujii K, Kanekura T. Comparison of oxidative stress on DNA, protein and lipids in patients with actinic keratosis, Bowen's disease and squamous cell carcinoma. The Journal of Dermatology. 2018;45(11):1319-23.

[26] Yamaba H, Haba M, Kunita M, Sakaida T, Tanaka H, Yashiro Y, et al. Morphological change of skin fibroblasts induced by UV Irradiation is involved in photoaging. Exp. Dermatol. 2016;25(Suppl 3):45-51.

[27] Benjamin CL, Ananthaswamy HN. p53 and the pathogenesis of skin cancer. Toxicology and Applied Pharmacology. 2007;224(3):241-48.

[28] Vaisman A, Woodgate R. Translesion DNA polymerases in eukaryotes: what makes them tick? Crit. Rev. Biochem. Mol. Biol. 2017;52(3):274-303.

[29] Matheus LT, Bolaños A. Micronúcleos: biomarcador de genotoxicidad en expuestos a plaguicidas. Revista de la Facultad de Ciencias de la Salud. Universidad de Carabobo. 2014;18(2):1826.

[30] Hoyos GL, Ramos AJ, Reyes CI. Evaluación de riesgo de cáncer en personas expuestas ocupacionalmente a solventes orgánicos. Rev Salud Pública. 2020;22(3):1-5.

[31] Zúñiga GG, Gómez MB, Zamora PA, Martínez GM, Bautista BM, Patiño VS, et al. Micronucleated erythrocytes in newborns rats exposed to three different types of ultraviolet-A (UVA) lamps from commonly uses devices. Journal of Photochemistry and Photobiology B: Biology. 2016; 165:141-46. doi: 10.1016/j.jphotobiol.2016.10.021. PMID: 27792890.

[32] Landrigan PJ, Espina C, Neira M. Global prevention of environmental and occupational cancer. Environmental Health Perspectives. 2011;119(7):a280-a281. doi:10.1289/ehp.1103871. PMID: 21719377 ; PMCID: PMC3222998. 